\title{
Meconium Plug Syndrome
}

National Cancer Institute

\section{Source}

National Cancer Institute. Meconium Plug Syndrome. NCI Thesaurus. Code C98980.

A transient colonic dysfunction in the newborn characterized by the delayed passage of meconium and associated intestinal dilatation. 